\title{
EXISTENCE OF SOLUTIONS OF FUNCTIONAL DIFFERENTIAL INCLUSIONS ${ }^{1}$
}

\author{
A. ANGURAJ and K. BALACHANDRAN \\ Bharathiar University \\ Department of Mathematics \\ Coimbatore $641 \quad 046$ \\ Tamil Nadu, INDIA
}

\begin{abstract}
We prove the existence of solutions of a functional differential inclusion. By using the variation of parameters formula we convert the functional differential inclusion into an integral inclusion and prove the existence of a fixed point of the set-valued mapping with the help of the Kakutani-Bohnenblust-Karlin fixed point theorem.
\end{abstract}

Key words: Set-valued maps, Functional differential inclusion, Integral inclusion, Kakutani's fixed point theorem.

AMS (MOS) subject classifications: $\quad 34 \mathrm{~A} 60,34 \mathrm{G} 20$.

\section{INTRODUCTION}

Fixed point theorems are widely used as a tool to prove the existence of solutions of differential inclusions. Schauder's fixed point theorem is used to prove the existence of solutions of differential inclusions [2] and functional differential inclusions with nonconvex right-hand side [8] in Banach spaces. Ky Fan's fixed point theorem is used in [7,10]. In [5], Marino used a fixed point theorem due to Martelli [6] for establishing the existence of solutions of a nonlinear differential inclusion in Banach spaces. Angell [1] obtained an existence theorem for integral inclusions of Urysohn type by using Kakutani-Bohnenblust-Karlin fixed point theorem. Papageorgiou [9] proved the same for the nonconvex case by using Schauder's fixed point theorem.

In this paper we prove the existence of solutions of functional differential inclusions via integral inclusions. First we convert the functional differential inclusion into an integral inclusion by using the variation of parameters formula. Then we use the Bohnenblust-Karlin extension of Kakutani's fixed point theorem [3] to prove the existence of solutions of the integral inclusion which is the solution of differential inclusion.

\footnotetext{
${ }^{1}$ Received: March, 1992. Revised: May, 1992.
} 


\section{BASIC IIYPOTIIESES}

Consider the differential inclusion

$$
\begin{gathered}
\dot{x}(t) \in L\left(t, x_{t}\right)+F\left(t, x_{t}\right), \text { a.e. on }[0, b] \\
x(t)=\phi(t) \text { on }[-r, 0]
\end{gathered}
$$

where $x_{t}:[-r, 0] \rightarrow R^{n}$ is continuous such that $x_{t}(\Theta)=x(t+\Theta)$.

In order to ensure the existence of solutions of the differential inclusion (1), we shall make the following assumptions:

(i) $\quad L:[0, b] \times L^{\infty}[-r, b] \rightarrow R^{n}$ is continuous and linear,

(ii) the set-valued map $F:[0, b] \times L^{\infty}[-r, b] \rightarrow 2^{R^{n}}$ is convex such that for any $(\bar{t}, \bar{\phi}) \in[0, b] \times L^{\infty}[-r, b]$,

$$
F(\bar{t}, \bar{\phi})=\bigcap_{\delta>0} c l \bigcup\{F(\bar{t}, \phi):\|\phi-\bar{\phi}\| \leq \delta\}
$$

that is, $F$ is upper semicontinuous in the sense of Kuratowski with respect to the variable $\phi$. Note that, as the intersection of closed sets each $F(t, \bar{\phi})$ is closed,

(iii) there exists a measurable set-valued function $P:[0, b] \rightarrow E^{1}$, a constant $M>0$, and for each $\epsilon>0$, a function $\Psi_{\epsilon} \in L^{\prime}\left([0, b] ; R^{n}\right), \Psi_{\epsilon}(t)>0$, such that for given $x \in L^{\infty}\left([-r, b] ; R^{n}\right)$ and selection $v(t) \in F\left(t, x_{t}\right)$, there exists a selection $\eta(t) \in P(t)$, with

$$
\int_{0}^{b} \eta(t) d t \leq M \text { and }|v(t)| \leq \Psi_{\epsilon}(t)+\epsilon \eta(t) .
$$

Let $f:[0, b] \times L^{\infty}[-r, b]$ be continuous and $C=C\left([-r, b] ; R^{n}\right)$. The variation of parameters formula for the initial value problem

$$
\begin{gathered}
\dot{x}(t)=L\left(t, x_{t}\right)+f\left(t, x_{t}\right), t \in[0, b] \\
x(t)=\phi(t) \text { on }[-r, 0]
\end{gathered}
$$

is given by [4]

$$
x_{t}=T(t, \sigma) \phi(\sigma)+\int_{\sigma}^{t} T(t, s) X_{0} f\left(s, x_{s}\right) d s
$$

where the operator $T(t, \sigma): C \rightarrow C$ is given by

$$
\begin{gathered}
T(t, \sigma) \phi=x_{t}(\sigma, \phi), \sigma \leq t \leq b \text { such that } \\
T(\sigma, \sigma)=I, T(t, \sigma) T(\sigma, s)=T(t, s)
\end{gathered}
$$


is a solution of the homogeneous equation

$$
\dot{x}(t)=L\left(t, x_{t}\right)
$$

and $X_{0}$ is defined by

$$
X_{0}(\Theta)=\left\{\begin{array}{cc}
0, & -r \leq \Theta<0 \\
I, & \Theta=0
\end{array}\right.
$$

where $I$ is the identity matrix.

Further we assume that

(iv) $T$ is a bounded linear operator, with bound $N$ and continuous as follows:

For $t^{\prime}, t^{\prime \prime} \in[0, b]$ and a $\delta>0$, there exists an $\epsilon>0$ such that

$$
\left|T\left(t^{\prime}, s\right)-T\left(t^{\prime \prime}, s\right)\right|<\epsilon \text { of }\left|t^{\prime}-t^{\prime \prime}\right|<\delta .
$$

Now we can write the equivalent form of the differential inclusion (1) as the integral inclusion

$$
\begin{gathered}
x(t) \in T(t, 0) \phi(0)+\int_{0}^{t} T(t, s) X_{0} F\left(s, x_{s}\right) d s, 0 \leq t \leq b \\
x(t)=\phi(t),-r \leq t \leq 0 .
\end{gathered}
$$

So in order to prove the existence of solutions of the differential inclusion (1), we have to prove the existence theorem for the integral inclusion (2). We prove this existence theorem by using Bohnenblust-Karlin extension of Kakutani's fixed point theorem.

Theorem 1: (Bohnenblust-Karlin) [3]

Let $\Sigma$ be a nonempty, closed convex subset of a Banach space $\mathscr{B}$. If $\Gamma: \Sigma \rightarrow 2^{\Sigma}$ is such that

(a) $\Gamma(\sigma)$ is nonempty and convex for each $\sigma \in \Sigma$,

(b) the graph of $\Gamma, \mathrm{g}(\Gamma) \subset \Sigma \times \Sigma$ is closed,

(c) $U\{\Gamma(\sigma) ; \sigma \in \Sigma\}$ is contained in a sequentially compact set $\mathcal{T} \subset \mathfrak{B}$, then the set-valued map $\Gamma$ has a fixed point, that is, there exists a $\sigma_{0} \in \Sigma$ such that $\sigma_{0} \in \Gamma\left(\sigma_{0}\right)$. 


\section{EXISTENCE}

Since our interest is to study the existence of the solutions of the differential inclusion (1), we will need to give a precise definition of the term solution.

Definition (1): A solution of the differential inclusion (1) is a function $x$, defined on $[-r, b]$ with $x_{0}(\Theta)=\phi(\Theta),-r \leq \Theta \leq 0$, and $\left.x\right|_{[0, b]} \in C\left([0, b] ; R^{n}\right)$, and such that there exists $v \in L^{1}\left([0, b] ; R^{n}\right)$ satisfying the inclusion $v(t) \in F\left(t, x_{t}\right)$ almost everywhere on $[0, b]$ and for which

$$
x(t)=T(t, 0) \phi(0)+\int_{0}^{t} T(t, s) X_{0} v(s) d s, 0 \leq t \leq b
$$

Now we may recast the initial-value problem as a problem for a fixed point of a setvalued mapping as follows; we introduce two set-valued mappings whose domain $S \subset L^{\infty}\left([-r, b] ; R^{n}\right)$ is defined by

$$
S=\left\{x \in L^{\infty}\left([-r, b] ; R^{n}\right):\left.x\right|_{[-r, 0]}=\phi,\left.x\right|_{[0, b]} \in C\left([0, b] ; R^{n}\right)\right\}
$$

Clearly $S$ is a closed convex set in $L^{\infty}\left([-r, b] ; R^{n}\right)$. We define the set-valued maps $\Phi: S \rightarrow L^{1}\left([0, b] ; R^{n}\right)$ and $\Psi: S \rightarrow 2^{S}$, respectively, by

$$
\Phi(x)=\left\{v \in L^{1}\left([0, b] ; R^{n}\right): v(t) \in F\left(t, x_{t}\right) \text { a.e. on }[0, b]\right\}
$$

and

$$
\Psi(x)=\left\{z \in S: z(t)=T(t, 0) \phi(0)+\int_{0}^{t} T(t, x) X_{0} v(s) d s, v \in \Phi(x),\left.z\right|_{[-r, 0]}=\phi\right\}
$$

Remark 1: Suppose that $x_{0} \in S$ is a fixed point of the mapping $\Psi$ defined by the relation (4), that is, suppose $x_{0} \in \Psi\left(x_{0}\right)$. Then $x_{0} \in S$ is a solution of the integral inclusion (2).

Theorem 2: [1]: Under the hypothesis (ii), for each $x \in S, \Phi(x)$ is not empty and the set $\Phi(S)$ defined by the relation (4) is an equi-absolutely integrable set and is weakly compact in $L^{1}\left([0, b] ; R^{n}\right)$.

Now we prove the relative compactness of the set $\Psi(S)$ and the convexity of $\Psi(x)$.

Theorem 3: Under hypotheses (iii) and (iv), for each $x \in S, \Psi(x)$ is not empty and the set $\Psi(S)$ defined by the relation (4) is a relatively sequentially compact subset of $L^{\infty}\left([0, b] ; R^{n}\right)$. 
Proof: First we prove that $\Psi(x)$ is not empty, for all $x \in S$. If we are given $x \in S$, then from Theorem $2, \Phi(x)$ is not empty. We choose $v \in \Phi(x)$ and define

$$
y(t)=T(t, 0) \phi(0)+\int_{0}^{t} T(t, s) x_{0} v(s) d s, 0 \leq t \leq b .
$$

Let $\epsilon>0$ be given and suppose that $\delta_{1}<\frac{\epsilon}{12 M N}$. Now for any $t^{\prime}, t^{\prime \prime} \in[0, b]$,

$$
\begin{aligned}
& \left|y\left(t^{\prime}\right)-y\left(t^{\prime \prime}\right)\right| \leq\left|T\left(t^{\prime}, 0\right)-T\left(t^{\prime \prime}, 0\right)\right||\phi(0)| \\
& +\int_{0}^{t^{\prime}}\left|T\left(t^{\prime}, s\right)-T\left(t^{\prime \prime}, s\right)\right|\left|X_{0}\right||v(s)| d s \\
& +\int_{t^{\prime}}^{t^{\prime \prime}}\left|T\left(t^{\prime \prime}, s\right)\right|\left|X_{0}\right||v(s)| d s \\
& \leq\left|T\left(t^{\prime}, 0\right)-T\left(t^{\prime \prime}, 0\right)\right||\phi(0)| \\
& \left.+\int_{0}^{t^{\prime}}\left|T\left(t^{\prime}, s\right)-T\left(t^{\prime \prime}, s\right)\right| \Psi_{\delta_{1}}(s)+\delta_{1} \eta(s)\right) d s \\
& \left.+\int_{t^{\prime}}^{t^{\prime \prime}}\left|T\left(t^{\prime \prime}, s\right)\right| \Psi_{\delta_{1}}(s)+\delta_{1} \eta(s)\right) d s \quad \text { (from (iii)) } \\
& \leq\left|T\left(t^{\prime}, 0\right)-T\left(t^{\prime \prime}, 0\right)\right||\phi(0)| \\
& +\int_{0}^{t^{\prime}}\left|T\left(t^{\prime}, s\right)-T\left(t^{\prime \prime}, s\right)\right| \Psi_{\delta_{1}}(s) d s+2 \delta_{1} N \int_{0}^{b} \eta(s) d s \\
& +N \int_{t^{\prime}}^{t^{\prime \prime}} \Psi_{\delta_{1}}(s) d s+\delta_{1} N \int_{0}^{b} \eta(s) d s \\
& \leq\left|T\left(t^{\prime}, 0\right)-T\left(t^{\prime \prime}, 0\right)\right||\phi(0)| \\
& +\int_{0}^{t^{\prime}}\left|T\left(t^{\prime}, s\right)-T\left(t^{\prime \prime}, s\right)\right| \Psi_{\delta_{1}}(s) d s+3 \delta_{1} M N \\
& +N \int_{t^{\prime}}^{t^{\prime \prime}} \Psi_{\delta_{1}}(s) d s
\end{aligned}
$$


Hence $\left|y\left(t^{\prime}\right)-y\left(t^{\prime \prime}\right)\right| \leq I_{1}+I_{2}+\frac{\epsilon}{4}+I_{3}$.

Now from the hypothesis (iv), there exists a $\delta_{2}>0$ such that $\left|T\left(t^{\prime}, 0\right)-T\left(t^{\prime \prime}, 0\right)\right|<\epsilon / 4|\phi(0)|$ if $\left|t^{\prime}-t^{\prime \prime}\right|<\delta_{2}$, that is, $I_{1}<\epsilon / 4$ if $\left|t^{\prime}-t^{\prime \prime}\right|<\delta_{2}$.

Also since $T$ is bounded and $\Psi_{\delta_{1}} \in L^{1}\left([0, b] ; R^{n}\right)$, then by using Lebesgue dominated convergence theorem, for a sufficiently small $\delta_{3}>0, I_{2}<\frac{e}{4}$ if $\left|t^{\prime}-t^{\prime \prime}\right|<\delta_{3}$.

Moreover, since $\Psi_{\delta_{1}}$ is integrable, we may conclude that there is a $\delta_{4}>0$ such that if $\left|t^{\prime}-t^{\prime \prime}\right|<\delta_{4}$, then

$$
\int_{t^{\prime}}^{t^{\prime \prime}} \Psi_{\delta}(s) d s<\frac{\epsilon}{4 N} \text { and therefore } I_{3}<\frac{\epsilon}{4}
$$

Therefore $\left|y\left(t^{\prime}\right)-y\left(t^{\prime \prime}\right)\right|<\frac{\epsilon}{4}+\frac{\epsilon}{4}+\frac{\epsilon}{4}+\frac{\epsilon}{4}=\epsilon$.

Hence the elements of $\Psi(S)$ restricted to the interval $[0, b]$ form an equicontinuous family.

Now choose $\delta<\min \left\{\delta_{2}, \delta_{3}, \delta_{4}\right\}$. Then the piecewise continuous function $z$ defined by

$$
z(t)=\left\{\begin{array}{cc}
\phi(t), & -r \leq t<0 \\
y(t), & 0 \leq t \leq b
\end{array}\right.
$$

lies in $S$. Hence $\Psi(x)$ is not empty.

To prove the theorem, it remains to show that $\Psi(S)$ is equibounded.

For a given $t_{0} \in[0, b]$

$$
\left|y\left(t_{0}\right)\right| \leq\left|T\left(t_{0}, 0\right)\right||\phi(0)|+\int_{0}^{t_{0}}\left|T\left(t_{0}, s\right)\right|\left|X_{0}\right||v(s)| d s .
$$

Taking $\epsilon=1$ in the hypothesis (iii), we have

$$
\left|y\left(t_{0}\right)\right| \leq N|\phi(0)|+N(M+K)<\infty
$$

since $\int_{0}^{b} \Psi_{1}(t) d t=K<\infty[1$, pp. 138].

Therefore $\Psi(S)$ is equibounded. Then by the Arzela-Ascoli theorem, any sequence $\left\{z_{k}\right\}$ in $\Psi(S)$ restricted to $[0, b]$ have a uniformly convergent subsequence. Hence the set $\Psi(S)$ is relatively sequentially compact.

Theorem 4: For each $x \in S$, the set $\Psi(x)$ defined by the relation (4) is convex. 
Proof: Let $y_{1}, y_{2} \in \Psi(x)$. Then there exists $v_{1}(t), v_{2}(t) \in F\left(t, x_{t}\right)$ such that

And so for $0<\lambda<1$, we have

$$
y_{i}(t)=T(t, 0) \phi(0)+\int_{0}^{t} T(t, s) X_{0} v_{i}(s) d s, \quad i=1,2 .
$$

$$
\lambda y_{1}(t)+(1-\lambda) y_{2}(t)=\int_{0}^{t} T(t, s) X_{0}\left[\lambda v_{1}(s)+(1-\lambda) v_{2}(s)\right] d s
$$

Since $F\left(t, x_{t}\right)$ is convex $\lambda v_{1}(t)+(1-\lambda) v_{2}(t) \in F\left(t, x_{t}\right)$ a.e. in $[0, b]$. Therefore $\lambda y_{1}(t)+(1-\lambda) y_{2}(t) \in \Psi(x)$.

Hence $\Psi(x)$ is convex.

Next we will prove that the graph of $\Psi, g(\psi)$ is closed. For that we use the following closure theorem.

Theorem 5:[1] Let $I=[0, b]$, consider the set-valued mapping $F: I \times L^{\infty} \rightarrow 2^{E^{n}}$ and assume that $F$ satisfies the hypothesis (ii) with respect to $\phi$. Let $v, v_{k}, x$ and $x_{k}$ be functions measurable on $I, x, x_{k}$ bounded, and let $v, v_{k} \in L^{1}\left(I ; R^{n}\right)$. Then if $v_{k}(t) \in F\left(t, x_{k_{t}}\right)$ a.e. in $I$ and $v_{k} \rightarrow v$ weakly in $L^{\prime}\left(I ; R^{n}\right)$ while $x_{k} \rightarrow x$ uniformly on $I$, then $v(t) \in F\left(t, x_{t}\right)$ a.e. in $I$.

Theorem 6: Under the hypotheses (ii), (iii) and (iv) the map $\Psi: S \rightarrow 2^{S}$ has a closed graph, that is,

$$
\{(x, y) \in S \times S: y \in \Psi(x)\} \text { is closed. }
$$

Proof: Let $\left\{x_{k}, y_{k}\right\}$ be a sequence of functions, $y_{k} \in \Psi\left(x_{k}\right)$, which converges to a limit point $(x, y)$ of $\mathcal{G}(\Psi)$. Thus $x_{k} \rightarrow x$ and $y_{k} \rightarrow y$ uniformly on $[0, b]$. We have to prove that $y \in \Psi(x)$.

By definition of $\Psi$, there exists a sequence $\left\{v_{k}\right\}$, with $v_{k} \in \Phi\left(x_{k}\right)$ such that

$$
y_{k}(t)=T(t, 0) \phi(0)+\int_{0}^{t} T(t, s) X_{0} v_{k}(s) d s .
$$

Without loss of generality, we may assume that $v_{k} \rightarrow v$ weakly in $L^{1}\left([0, b] ; R^{n}\right)$ and from Theorem $5, v(s) \in F\left(s, x_{s}\right)$.

To prove $y \in \Psi(x)$ we wish to show that $y$ satisfies the equation

$$
y(t)=T(t, 0) \phi(0)+\int_{0}^{t} T(t, s) X_{0} v(s) d s
$$


which, for convenience, we will write symbolically as

$$
y-v=0 .
$$

Recognizing that $\left(y_{k}, v_{k}\right)$ satisfying the above relation, we may write

$$
|y-v| \leq\left|y-y_{k}\right|+\left|v_{k}-v\right| \text {. }
$$

It is enough to show that the relation (5) holds pointwise.

Let us fix $t_{0} \in[0, b]$. Since $\left\{y_{k}\right\}$ uniformly converges to $y$, we have that $\left|y\left(t_{0}\right)-y_{k}\left(t_{0}\right)\right|<\epsilon / 2$.

Also since $T$ is bounded in $\left(L^{1}\left([0, b] ; R^{n}\right)^{\prime}\right.$ and $\left\{v_{k}\right\}$ weakly converges in $L^{1}\left([0, b] ; R^{n}\right)$,

$$
\left|v_{k}\left(t_{0}\right)-v\left(t_{0}\right)\right|<\frac{\epsilon}{2}
$$

Therefore given $\epsilon>0$,

$$
\left|y(t)-T(t, 0) \phi(0)-\int_{0}^{t} T(t, s) X_{0} b(s) d s\right|<\frac{\epsilon}{2}+\frac{\epsilon}{2}=\epsilon .
$$

Hence $y \in \Psi(x)$ and therefore $(x, y) \in \mathcal{G}(\psi)$, that is the graph of $\Psi$ is closed. And the proof is complete.

So far we have verified that all of the hypotheses of Theorem 1 are satisfied. We may thus consider the following existence theorem.

Theorem 7: Under the hypotheses (ii) - (iv) the set-valued map $\Psi: S \rightarrow 2^{S}$ has a fixed point in $S$; consequently, the integral inclusion (2) has a solution in $S$.

Since the existence of solution to the integral inclusion (2) is equivalent to the existence of solution to the differential inclusion (1), we state our main theorem.

Theorem 8:Under the hypotheses (i)-(iii), the differential inclusion (1) has a solution.

\section{REFERENCES}

[1] T.S. Angell, "Existence of multivalued Urysohn integral equations", Journal of Optimization Theory and Applications, Vol. 46, pp. 129-150, (1985).

[2] J.P. Aubin and A. Cellina, "Differential Inclusions”, Springer, Berlin, 1984.

[3] H.F. Bohnenblust and S. Karlin, "On a Theorem of Ville", Contributions to the Theory of Games: I, Edited by H.W. Kuhn and A.W. Tucker, Princeton University Press, Princeton, New Jersey, pp. 155-160, (1950). 
[4] J.K. Hale, "Theory of Functional Differential Equations", Springer-Verlag, New York, 1977.

[5] G. Marino, "Nonlinear boundary value problems for multivalued differential equations in Banach spaces", J. of Nonlinear Analysis: Theory, Methods and Applications, Vol. 14, pp. 545-558, (1990).

[6] M. Martelli, "A Rothe's type theorem for noncompact acyclic-valued maps", Boll. U.M.I., Vol. 4, pp. 70-76, (1975).

[7] N.S. Papageorgiou, “Boundary value problems for evolution inclusions”, Commentat. Math. Univ. Carol., Vol. 29, pp. 355-363, (1988).

[8] N.S. Papageorgiou, "Functional differential inclusions in Banach spaces with nonconvex right-hand side", Funckcialaj Ekvacioj, Vol. 32, pp. 145-156, (1989).

[9] N.S. Papageorgiou, "Existence of solutions for integral inclusions of Urysohn type with nonconvex-valued orientor field", Journal of Optimization Theory and Applications, Vol. 64, pp. 207-215, (1990).

[10] P. Zecca and P.L. Zezza, "Nonlinear boundary value problems in Banach space for multivalued differential equations in a noncompact interval", J. of Nonlinear Analysis: Theory, Methods and Applications, Vol. 3, pp. 347-352, (1979). 


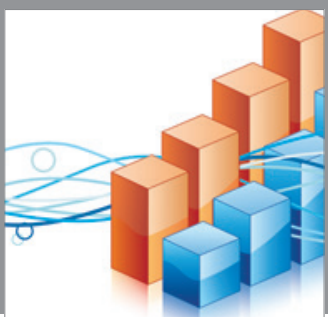

Advances in

Operations Research

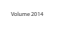

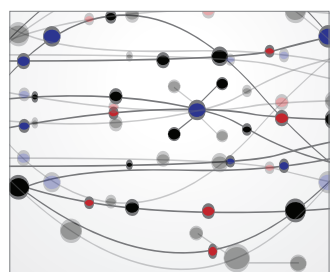

\section{The Scientific} World Journal
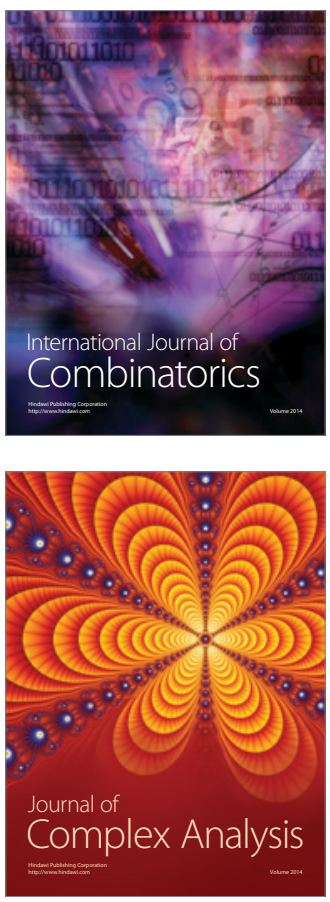

International Journal of

Mathematics and

Mathematical

Sciences
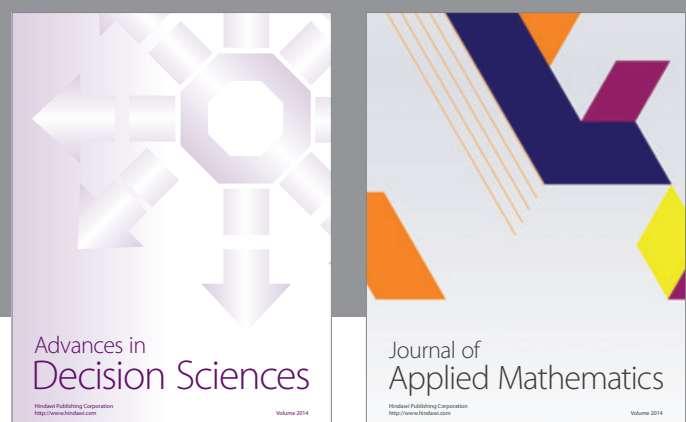

Journal of

Applied Mathematics
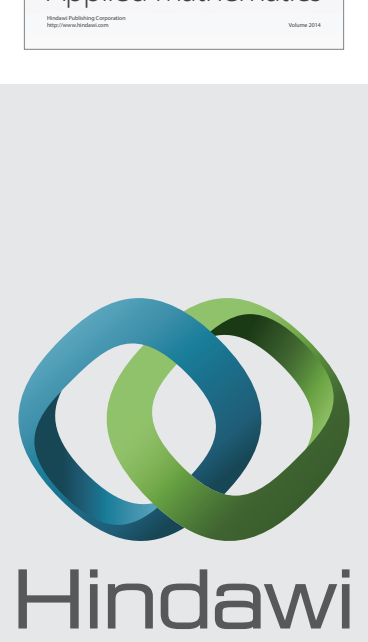

Submit your manuscripts at http://www.hindawi.com
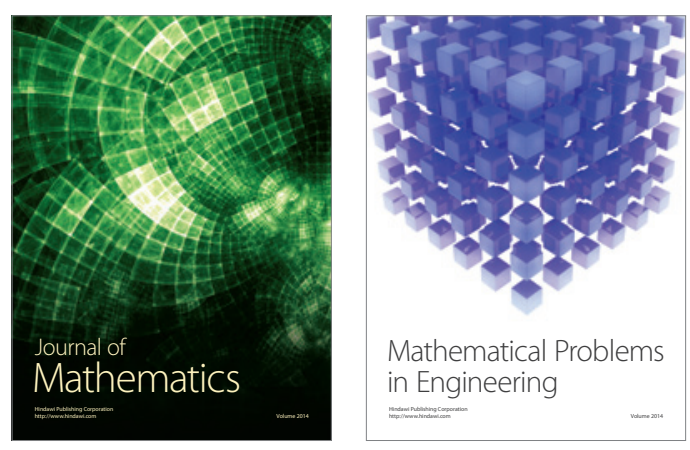

Mathematical Problems in Engineering
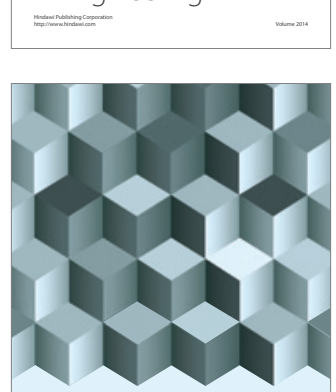

Journal of

Function Spaces
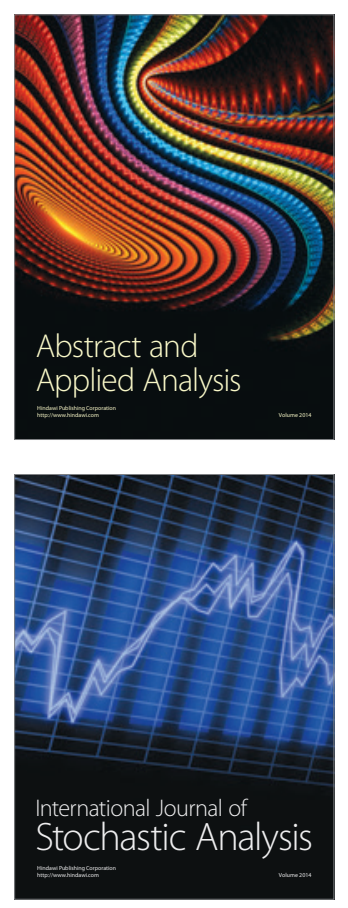

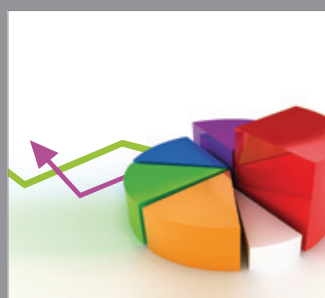

ournal of

Probability and Statistics

Promensencen
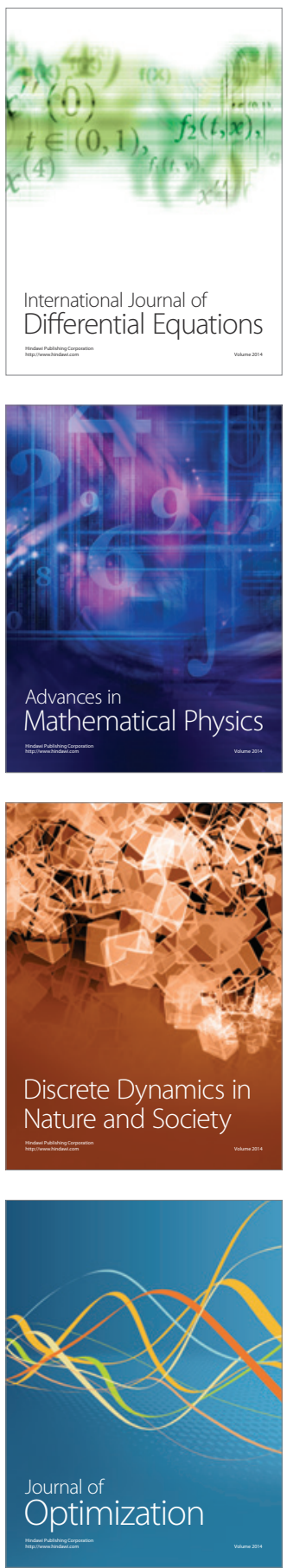